\title{
Genetic and structural alterations of enamel and dentin- amelogenesis imperfecta, dentinogenesis imperfecta and dentin dysplasia
}

\begin{abstract}
Genetic alterations of enamel and dentin include different sub-groups recognized on the basis of their clinical appearance. Ameloblasts secrete three major enamel ECM proteins: AMEL (amelogenin associated with Amelogenesis Imperfecta phenotypes, ranging from hypoplastic to hypomineralized enamel), AMBN (ameloblastin) and ENAM (enamelin) They are localized within a cluster of genes critical to biomineralization mapped on chromosome 4q21. Hypoplastic enamel displays secretory defects (pitted, rough or local). Hypomineralized (with eruption pathology), hypocalcified types with mineralization defects, and hypomature enamel result to altered protein processing and crystallite maturation defects. They display a chalky appearance, orange, brown or white colour. Enamel is pigmented, snow capped. Dentin defects are classified into three types of Dentinogenesis Imperfecta (DGI, types I-III) and two types Dentin Dysplasia (DDs, types I and II). DGI type II was originally called hereditary opalescent dentin or Capdepont's teeth. Clinically, DGI-II is characterized by soft blue-brown, translucent teeth (opalescent teeth). Abnormal dentin obliterate the pulp chamber of DD type I. Genetically altered enamel and dentin structures allow significant insigths on dental tissue genetic alterations, and consequently increase our understanding of the formation of normal dental tissues. The affected dental tissues involve gene mutations, translated into structural proteins and/ or implicated in the composition of dental tissues. This shed light on the cleavage of the constituent molecules of the ECM.
\end{abstract}

Keywords: proteins, molecules, phenotypes, dental tissues, gene mutations, heterogeneity, clinical appearance, autosomal recessive, basement membrane, maturation stage, enamel crystallites, gene
Volume 10 Issue 4 - 2019

\section{Michel Goldberg}

Department of Oral Biology, Faculty of Fundamental and Biomedical Sciences, Paris Descartes University, France

Correspondence: Michel Goldberg, Professor Emeritus, Department of Oral Biology, Paris Descartes University, Faculty of Fundamental and Biomedical Sciences \& INSERMUMR-S I 24. Stem cells, signalization and prions. 45 rue des saints pères, 75006 , Paris, France,Tel 33162676709, Email mgoldod@gmail.com

Received: June 4, 2019 | Published: August 28, 2019
Abbreviations: ECM, extracellular molecules; DD, dentin dysplasia; DI, dentinogenesis imperfecta; XLR, X-linked recessive; DSPP, dentin sialophosphoprotein; AR, autosomal recessive; AD, autosomal dominant

\section{Introduction}

The protein gene family includes extracellular molecules (ECM) proteins, responsible for dentin/bone coding (DSPP, DMP1, IBSP, $M E P E$, and $S P P 1$ ), enamel (AMEL, ENAM, $A M B N$, and $A M T N$ ), as well as milk casein, and some salivary protein genes (Table 1). These molecules encompass inherited defects of dental enamel (AI) and dentin (DI and DD). They display both clinical and genetic heterogeneity. These groups include different sub-types recognized on the basis of their clinical appearance. Diseases affecting tooth structures have been classified into distinct tissues [enamel (AI) versus dentin (DI \& DD), the specificity of the mutation (syndromic versus non-syndromic), and their pattern of inheritance [autosomal dominant (AD), autosomal recessive (AR), or X-linked recessive (XLR)]. Mutations in the AMELX , ENAM , MMP20 and KLK4 genes are associated with specific AI types. Another series of gene mutations influence dentin structure and composition [dentinogenesis imperfecta (DI) and dentin dysplasia (DD)]. These mutated genes are implicated in defective dental tissues. ${ }^{1-3}$

Table I SCPP genes and ancestors

\begin{tabular}{lll}
\hline Gene symbol & Protein name & $\begin{array}{c}\text { Protein } \\
\text { distribution }\end{array}$ \\
\hline Ancestor & secreted protein, acidic, cysteine rich (osteonectin) & skeleton \\
SPARC & $\begin{array}{l}\text { secreted protein, acidic, cysteine-rich like I protein } \\
\text { (high endothelial venule protein) }\end{array}$ & brain \\
SPARCLI & dentin sialophosphoprotein & \\
SCPP & dentin matrix acidic phosphoprotein I & dentin, bone \\
DSPP & integrin-binding sialoprotein (bone sialoprotein) & dentin, bone \\
DMPI & matrix extracellular phosphoglycoprotein & dentin, bone \\
IBSP & & \\
MEPE &
\end{tabular}


Table Continued...

\begin{tabular}{|c|c|c|}
\hline Gene symbol & Protein name & $\begin{array}{l}\text { Protein } \\
\text { distribution }\end{array}$ \\
\hline SPPI & secreted phosphoprotein I (osteopontin) & dentin, bone \\
\hline AMEL & amelogenin & enamel \\
\hline ENAM & enamel & enamel \\
\hline AMBN & ameloblastin (sheathlin, amelin) & enamel \\
\hline AMTN & (UNQ689) & enamel \\
\hline ODAM & odontogenic, ameloblast associated (APIN protein) & $\begin{array}{l}\text { milk, saliva, } \\
\text { enamel }\end{array}$ \\
\hline FDCSP & follicular dendritic cell secreted peptide & milk, saliva, PDL \\
\hline MUC7 mucin 7 & & saliva \\
\hline $\begin{array}{l}\text { PROLI proline-rich I (basic proline- } \\
\text { rich lacrimal protein I) }\end{array}$ & & saliva \\
\hline $\begin{array}{l}\text { PROL3 proline rich } 3 \text { [submaxillary } \\
\text { gland androgen-regulated protein } 3\end{array}$ & & \\
\hline homolog B (mouse)] & & saliva \\
\hline $\begin{array}{l}\text { PROL5 (SMR3A) proline rich } 5 \\
\text { [submaxillary gland androgen- } \\
\text { regulated protein } 3\end{array}$ & & saliva \\
\hline homolog A (mouse)] & & \\
\hline LOC40II37 & & saliva \\
\hline HTNI histatin I & & saliva \\
\hline HTN3 histatin 3 & & saliva \\
\hline STATH statherin & & saliva \\
\hline CSN3 & k-casein & milk \\
\hline CSN2 & b-casein & milk \\
\hline CSNISI & aSI-casein & milk \\
\hline \multicolumn{3}{|l|}{$\begin{array}{l}\text { PDL = Periodontal ligament; } \\
\text { LOC } 40 \text { I I } 37 \text { = the locus symbol } \\
\text { given in the genome sequence } \\
\text { database. }\end{array}$} \\
\hline $\begin{array}{l}\text { Many salivary SCPPs are also } \\
\text { present in tears. (reprinted from 4) }\end{array}$ & & \\
\hline
\end{tabular}

\section{Amelogenin imperfecta}

In mammals, ameloblasts secrete three major enamel ECM proteins: AMEL (amelogenin associated with AI phenotypes, ranging from hypoplastic to hypomineralized enamel), AMBN (ameloblastin) and ENAM (enamelin localized within a cluster of genes critical to biomineralization, mapped on chromosome 4q21). Mutations result in enamel hypoplasia. In addition, AMTN (amelotin) is preferentially expressed by ameloblasts, in the incisor and molar basement membranes, but exclusively during the late maturation stage. ${ }^{4,5}$

Mutations of MMP20 and KLK4 are proteinases critical for processing enamel matrix components. They are located on chromosomes 11q23 and 19q13 respectively., ${ }^{6,7}$ In humans, enamel defects are including several types of AI, leading to enamel hyploplasia or hypomineralization. They are also known as hereditary enamel dysplasia, hereditary brown enamel, or hereditary brown opalescent teeth. ${ }^{8-12} \mathrm{AI}$ is an heterogeneous group charcterized by defects in the formation of enamel due to mutations in AMELX (14 X-linked AI, AIH1), and/or in ENAM (5 autosomal-dominant AI, and AIH2 genes). More than 50 mutations have been identified, based upon the phenotypes and the mode of inheritance.

In enamel, three main groups have been reported: ${ }^{8-12}$

1. Hypoplastic enamel (secretory defects- pitted, rough or local) (mapped to human chromosome 4q11-q21), hypoplasia of the enamel layer. Enamel is thin but mineralized. Pitted enamel localized or diffuse, smooth or rough.

2. Hypomineralized (with eruption pathology) or hypocalcified types (with mineralization defects). This pathology is caused by maturation failure. Enamel is of full thickness but is weak. They are further subdivided into hypomaturation and hypocalcified AI. Incomplete removal of protein from the enamel matrix produces brittle enamel, whereas insufficient 
transport of calcium ions into the developing enamel produces soft enamel (diffuse hypocalcification).

3. Hypomature (protein processing and crystallite maturation defects - chalky appearance, orange, brown or white color). Enamel is diffuse and pigmented, snow capped.

$\mathrm{X}$-linked alterations in the human amelogenin gene (AMELX) have been already reported. ${ }^{7}$

1. Amelogenin (AMELX, Xp22.3) is the most abundant enamel matrix protein. Mutational analyses have been carried out They constitute a group of over twenty AMELX mutations, including large deletions, nonsense and missense variants. Heterozygous mutations tend to present in female AI patients stripes of normal and AI affected enamel. In male, the defect is determined by the type and position of AMELY on the Y chromosome.

2. Ameloblastin (AMBN, 4q13.3) have shown that cleavage products accumulate in the sheath space separating rod and interrod enamel, maintaining rod boundaries.

3. Enamelin (ENAM, 4q13.3). Immediately cleaved after secretion, this molecule plays a role in crystal elongation. AMELX and ENAM mutations cause X-linked and autosomal dominant AI, respectively.

4. During the maturation stage, enamel proteins are degraded and removed. The enamel layer hardens as the crystallites grow in width and thickness. MMP20 and KLK4 mutations cause autosomal recessive pigmented hypomaturation AI, which is characterized by the retention of enamel proteins and a reduction in enamel hardness. ${ }^{6,7}$ Eleven missense, nonsense, frameshift and splice site mutations have been described. KLK4 encode a serine protease expressed and secreted both during the transition and maturation stages of amelogenesis. ${ }^{13-15,16}$

The classification of the IV types of AI was revisited. ${ }^{8,15,16}$ Now, this classification includes the following phenotypes:

1. Hypoplastic Type I, (chacterized by insufficient appositional growth and an associated crystal elongation, leaving the enamel layer pathologically thin, or hypoplastic). The most severe form of hypoplastic AI is enamel agenesis (where there is almost no clinical or radiographic evidence of enamel). Teeth are yellowish, with a rough structure, with a pitted enamel, from white to yellow-brown, often arranged in rows and columns, or pits and groves in a horizontal fashion across the middle third of tooth.

2. Hypomatured type II, is of normal thickness but has a mottled appearance, with crystallites displaying defective mineralization. Serine proteases play a critical role in most tissues. It includes the KLK4 gene family. Abnormal KLK4 activity influences the growth of enamel crystallites, reducing their thickness and/or width. Enamel is therefore incompletely mineralized. ${ }^{16}$

3. Hypocalcified type III: The mutation is located in the homeobox gene DLX3 positioned on the chromosome locus 17q21. This mutation is at the origin of the tricho-dento-osseous syndrome, characterized by enamel hypoplasia.

4. Hypoplastic-hypomaturation type IV that is a mixture of hypomaturated- hypoplastic forms associated with taurodontism. These forms of AI (amelogenesis imperfecta) are associated with various forms of taurodontism (AIHHT: DLX3) (Figure $1 \&$ Table 2).

\section{CLASSIFICATION OF TAURODONTISM}

\section{CYNODONT HYPODONT MESODONT HYPERDONT PYRAMIDAL}

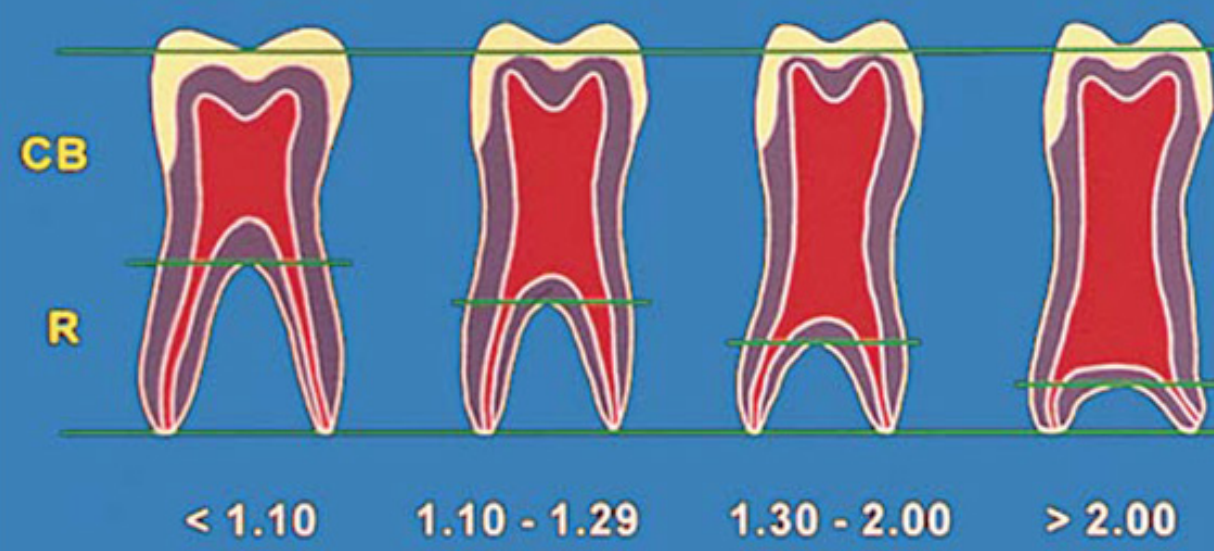

CB:R

Figure I Classification of taurodontism type IV AI. 
Table 2 Classification of $\mathrm{Al}$ (according to ${ }^{7,12}$ )

Type I Hypoplastic
Type II Hypomaturation
Type III Hypocalcified
Type IV: Hypomaturation-hypoplastic with taurodontism

Mutation of the gene encoding $M M P$-20 (enamelysin) is located in the intron 6 splice acceptors. Enamel is pigmented, with a mottled rough surface. ${ }^{10}$ During development and mutations, the kallikrein 4 (KLK4) and enamelysin (MMP20) genes cause autosomal recessive AI. ${ }^{6,7}$ Integrin, 6 is a member of a large family of cell-surface-adhesion receptors facilitating interactions with the cytoskeleton. ${ }^{14}$

Four enamelin gene (ENAM) defects have been identified in order to gain information related to genotype/phenotype correlations. The IVS6-2A $<$ C mutation exhibits horizontal groves of hypoplastic enamel. In g.8344delG mutation, a generalized hypoplastic enamel is observed with shallow horizontal groves in the middle $1 / 3$ of anterior teeth. ${ }^{8,9}$
Laminin (LAMA3), and LAMB3 mutation are also at the origin of amelogenesis imperfecta, ${ }^{12}$ including the mutation in the last exon of LAMB3. Enamel formation is particularly sensitive to defects in hemi-desmosome/basement membrane complexes. The syndromic and non-syndromic forms of AI are etiologically related to this mutation.

Laminin (formerly laminin V) is a component of basement membranes and comprised 3 subunits. Laminin is anchored to epithelial cells by collagen XVII and activates integrin signaling through $\alpha 6 / \beta 4$ receptors. ${ }^{11-13}$ AMTN (Amelotin) is a proline, leucine, threonine and glutamine-rich protein, secreted during the transition and maturation stage of ameloblasts. The molecule bind to itself, to ODAM (odontogenic, ameloblast-associated) and to SCPPPQ1 (secretory calcium-binding phosphoprotein-glutamine-rich 1 ). ${ }^{5}$ COL17A1 is expressed throughout enamel formation.

Non-syndromic AI-causing mutations have been found in genes encoding secreted enamel matrix proteins and proteases (AMELX, ENAM, C4orf26, MMP20, KLK417-(12-14), intracellular (FAM83H, WDR72), transmembrane (SLC24A4, COL17A1), and basement membrane (LAMA3, LAMB3) proteins (Figure 2) ${ }^{15,16-19}$

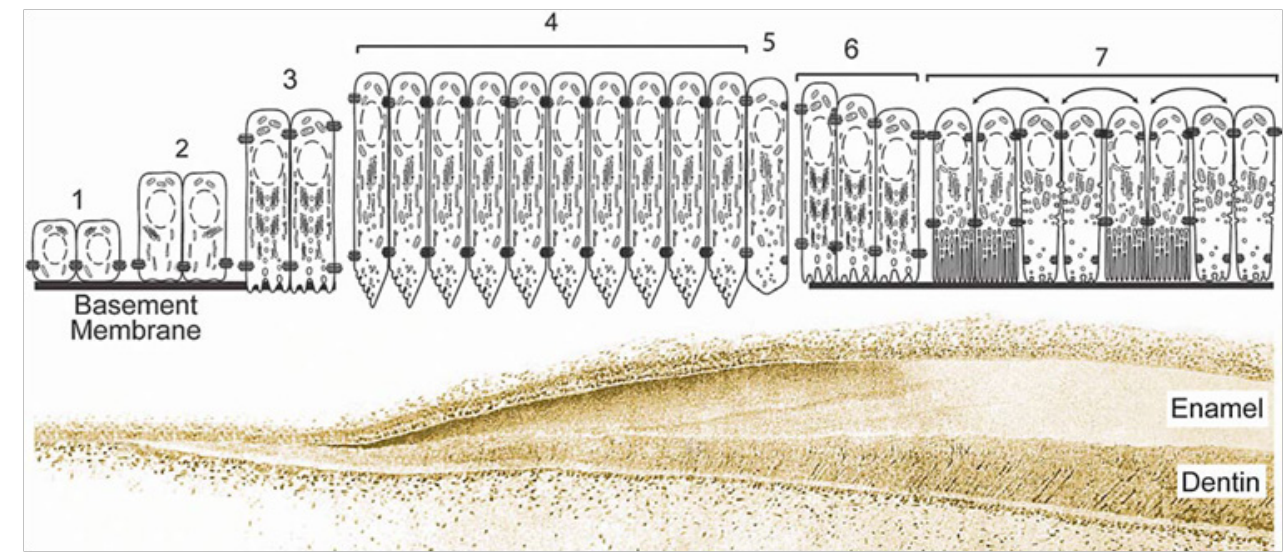

Figure 2 Reprinted from. ${ }^{13}$

\section{Dentinogenesis imperfecta (DGI) I 7-2I}

Dentin defects are classified into three types of DI (DGI, types I-III) and two types DD (DDs, types I and II). ${ }^{20,21}$ In DGI, the dentin is defective and soft. In DD, the pulp chambers may be obliterated by abnormal dentin. Mutations of DSPP cause DD type II. They have been mapped to chromosome 4q21. The incidence of DI is evaluated between $1 / 6000$ and $1 / 8000$ children.

The known mutations of collagen genes and their phenotypic effects in OI is increasing, but so far, no definitive relation has been established between the type of OI and the manifestations of the dental defects.

1. Clinical features associated with DGI type I (and DD) include joint hyperextensibility, short stature, hearing loss and sclera brown-blue or opalescent, and brown appearance of teeth..$^{18,19}$ Crowns appear bulbous. Pulp chambers are small or obliterated. Roots are narrow with small or obliterated canals. Defective formation of dentin-specific phosphophoryn. The defective gene has been mapped to the mouse $5 \mathrm{q} 21$, corresponding to the human 4q21 locus. It is a rootless teeth. Dmp1 gene is a candidate for type II DI. Type I is the defect associated with osteogenesis imperfecta.

2. DGI type II was originally called hereditary opalescent dentin or Capdepont's teeth.. Clinically, DGI-II is characterized by soft blue-brown, translucent teeth (opalescent teeth). Deciduous teeth are usually more affected than permanent teeth, although both primary and secondary dentitions display structural abnormalities. The teeth have short constricted roots and dentin hypertrophy leading to pulpal obliteration before or just after eruption. The two genes encoding type I collagen display missense mutations. The incidence of DI type II is between $1 / 6000$ and $1 / 8000$. The primary dentition are more affected than the permanent teeth. The mutation found is the dentin sialophosphoprotein (DSPP), gene, located on chromosome 4q22.1. The structure of the gene consists of 5 exons. Tooth anomalies of number, whereas tooth agenesis hypo/oligodontia results from the mutation of the homeobox gene MSX1. The absence of most permanent teeth with or without hypodontia in primary teeth are resulting from PAX9 mutations. ${ }^{15}$ Osteogenesis is not a constant feature. Bulbous crowns are typical with marked cervical constriction. Hearing may be lost.

3. DGI type III initially described as the triracial isolate in Brandywine Maryland. DI mutation have large pulp chambers, gradually obliterated (1:15). As shell teeth with a wide pulp chamber, the DGI type III results from mutation of DSPP. Mapped to chromosome 4q, it may be the same as in type II DI. Osteogenesis imperfecta is linked to DI and/or DD (Figure 3)..$^{20,21}$ 


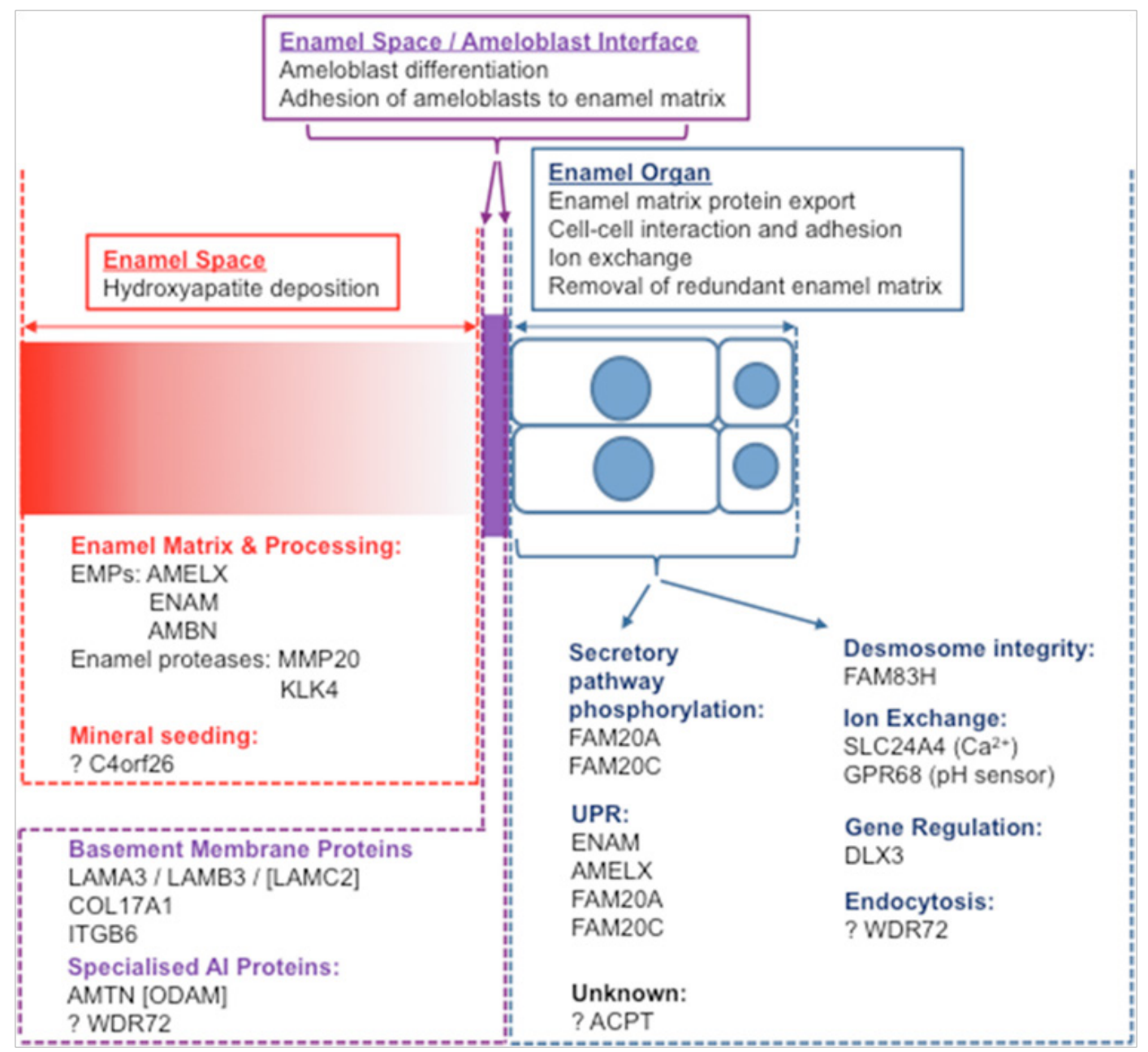

Figure 3 According to. ${ }^{19}$

\section{Dentin dysplasia (DD): genes involved in dentin dysplasia formation}

DD type I (DD-1) : Abnormal dentin obliterate the pulp chambers of DD type I. The prevalence of type I and II DD is about 1/100 000 . They have short roots. Early exfoliation and periapical radiolucencies are noted. DD type II is detectable in deciduous teeth but not in permanent teeth. They have large pulpolithes (denticles or pulp stones) within the pulp. The SIBLING family of proteins includes dentin sialophosphoprotein (DSPP), osteopontin, IBSP (integrin binding sialoprotein), $D M P-1$ and $M E P E$ (matrix extracellular phosphoglycoprotein). They display genes that are clustered on chromosome 4q. DSPP is located at 4q22.1 and consists of 5 exons. Three distinct protein products are formed from the initially translated polypeptide (DSPP): DSP, DGP and DPP. Dentin sialoprotein (DSP) and dentin phosphoprotein (DPP) overexpression are produced by the cleavage of a single gene ( $D S P P)$. After an initial cleavage producing the release of DPP, MMP20 generate DSP and DGP. Immediately after cleavage, DPP moves to the mineralization front and binds to collagen. DGP contains 4 phosphorylated serines and one N-glycosylated asparagine. The clinical phenotypes associated with DSPP mutations appear to represent a continuum of phenotypes. Thus, these disorders should be called DSPP-associated dentin defects, with DD type II representing the mild end of the phenotypic spectrum and DI type III expressing the critical end.
Dentin dysplasia type I (DD-1) is usually associated with osteogeniesis imperfecta. It is a consequence of the disintegration of Hertwig's epithelial root sheath and the subsequent migration of epithelial cells to the dental papilla. It regulates the induction of synthesis of dentin matrix. The root formation is defective. The pulp chambers of deciduous teeth are completely obliterated, whereas a crescent-shaped pulpal remnant persists in the permanent teeth. CollA1 and CollA2 are encoded by the COL1 A1 and COL1 A2 loci at $17 \mathrm{q} 21.3-\mathrm{q} 22$ and $7 \mathrm{q} 22.1$, respectively.

Dentin dysplasia type II (DD-2) or hereditary opalescent dentin: the abnormal dentin matrix has been reported to stain positively for reticulin, suggesting the presence of type III collagen. Abnormalities of noncollagenous components include changes in the amount of different glycosaminoglycans and the presence of fibronectin. The deficiency in dentin phosphoprotein is common in types I and II DI. The pulp chambers of deciduous teeth are completely obliterated. In permanent teeth, denticles are found. ${ }^{20,21}$

To conclude, genetically altered enamel and dentin structures allow significant insigths on dental tissue abnormalities, and consequently on our understanding of the formation of normal dental tissues. The affected tissues (enamel, dentin and pulp) involve gene mutations, consequently translated into structural proteins and/or implicated in the composition of dental tissues. ${ }^{12,15-17}$ This enlighten also the cleavage of the constituent molecules of the ECM (Figure 4). 


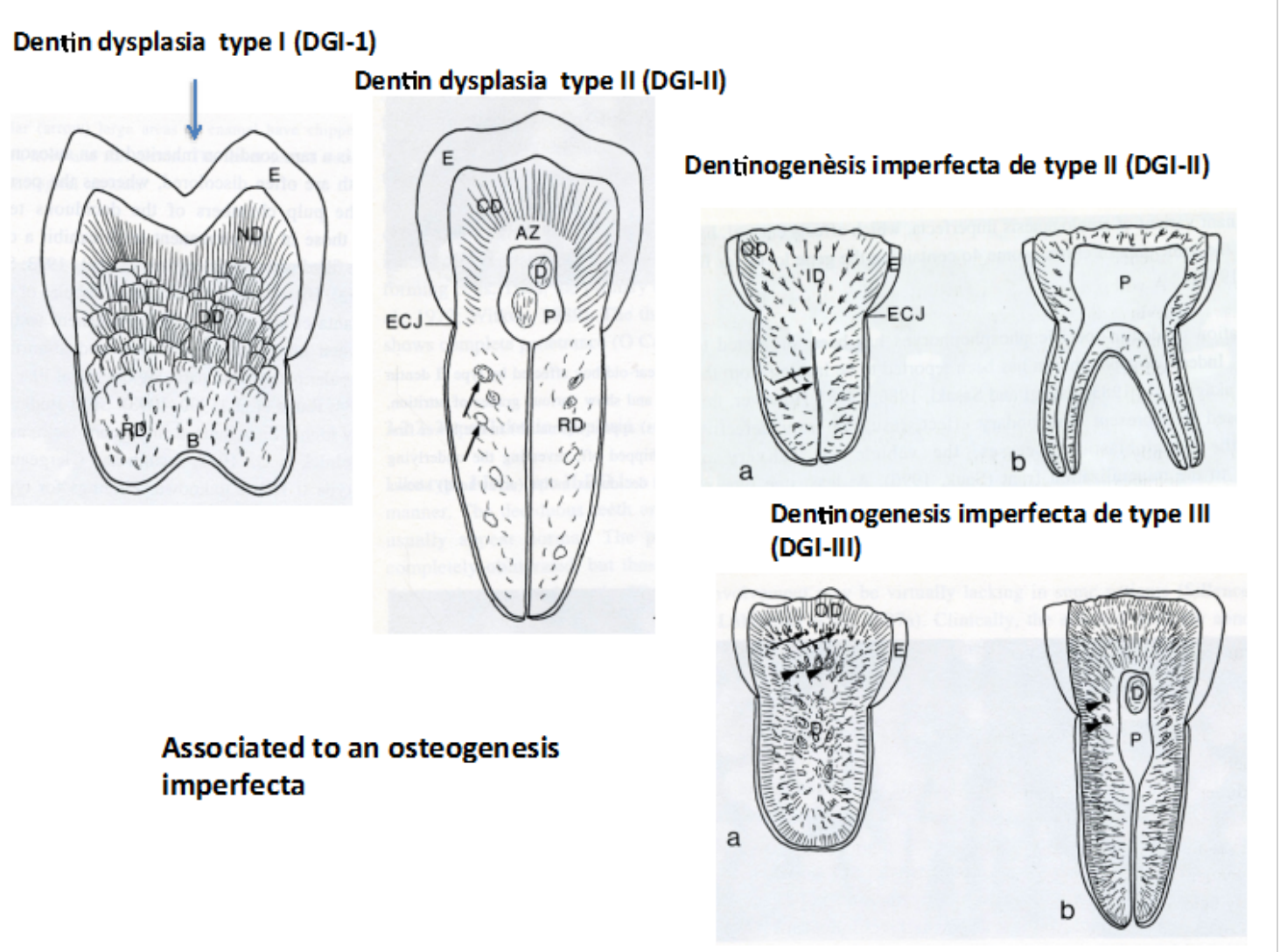

Figure 4 Reproduced from 20, 21 .

\section{Funding details}

None.

\section{Acknowledgments}

None.

\section{Conflict of interest}

The authors declare that there is no conflict of interest.

\section{References}

1. Wright JT, Hart TC, Hart PS, et al. Human and mouse enamel phenotypes resulting from mutation or altered expression of $A M E L, E N A M, M M P 20$ and KLK4. Cells Tissues Organs. 2009;189:224-229.

2. Wright JT, Torain M, Long K, et al. Amelogenesis Imperfecta : genotypephenotype studies in 71 families. Cells Tissues Organs. 2011;194(24):279-283.

3. Hart PS, Hart TC. Disorders of human dentin. Cells, Tissues, Organs. 2007;186(1):70-77.

4. Kawasaki K, Buchanan AV, Weiss KM. Gene duplication and the evolution of vertebrate skeletal mineralization. Cells Tissues Organs. 2007;186(1):7-24.

5. Moffat P, Smith CE, StArnaud D, et al. Cloning of rat amelotin and localization of the protein to the basal lamina of maturation stage ameloblasts and junctional epithelium. Biochem J. 2006;39(1):37-46.

6. Papagerakis P, Lin H-K, Lee KY, et al. Premature stop codon in MMP20 causing Amelogenesis Imperfecta. J Dent Res. 2008;87(1):56-59.
7. Hart PS, Hart TC, Michalec MD, et al. Mutation in kallikrein 4 causes autosomal recessive hypomaturation amelogenesis imperfecta. $\mathrm{J}$ Med Genet. 2004;41(7):545-549.

8. Witkop CJ Jr. Amelogenesis imperfecta, dentinogenesis imperfecta and dentin dysplasia revisited: problems in classification. J Oral Pathol. 1989;17(9-10):547-553.

9. Kim JW, Simmer JP, Hu YY, et al. Amelogenin p.M1T and p.W4S mutations underlying hypoplastic X-linked amelogenesis imperfecta. $J$ Dent Res. 2004;83(5):378-383.

10. Kim JW, Seymen F, Lin BP, et al. ENAM mutations in autosomaldominant amelogenesis imperfecta. J Dent Res. 2005;84(3):278-282.

11. Kim JW, Simmer JP, Hart TC, et al. MMP-20 mutation in autosomal recessive pigmented hypomaturation amelogenesis imperfect. $\mathrm{J}$ Med Genet. 2005;42(3):271-275.

12. Hu JCC, Chun YHP, Al Hazzazzi T, et al. Enamel formation and amelogenesis imperfecta Cell Tissue Organs. 2007;186(1):78-85.

13. Uchida T, Tanabe T, Fukae M, et al. Immunocytochemical and immuno chemical detection of a $32 \mathrm{kDa}$ nonamelogenin and related proteins in porcine tooth germs. Arch Histol Cytol . 1991;54:527-538.

14. Kim JW, Seymen F, Lee KE, et al. LAMB3 mutations causing autosomaldominant Amelogenesis Imperfecta. J Dent Res. 2013;92(10):899-904.

15. Bailleul-Forestier I, Molla M, Verloes A, et al. The genetic basis of inherited anomalies of the teeth. Part 1 : Clinical and molecular aspects of non-syndromic dental disorders. Eur J Med Genet. 2008;51(4):273-291.

16. Witkop CJ Jr. Manifestations of genetic diseases in the human pulp. Oral Surg Oral Med Oral Pathol. 1971;32(2):278-316. 
17. Ranta H, Lukinmaa PL, Waltimo J. Heritable dentin defects: nosology, pathology, and treatment. Am J Medical Genetics. 1993;45(2):193-200.

18. Shields E, Bixler D, El-Kafrawy A. A proposed classification for heritable human dentine defects with a description of a new entity. Arch Oral Biol. 1973;18(4):543-553.

19. Smith CEL, Poulter JA, Antanaviciute A, et al. Amelogenesis Imperfecta : genes, proteins, and pathways. Frontiers in Physiology. 2017;8:1-22.
20. Waltimo J, Ranta H, Lukinmaa PL. Transmission electron microscopic appearance of dentin on type II dentin dysplasia. Scand J Dent Res. 1991;99(5):349-356.

21. Waltimo J. Developmental defects of human dentin matrix- an ultrastructural study. Academic Dissertationn University of Helsinki. 1996. 